\title{
A PROBLEM OF FINITE BENDING OF TOROIDAL SHELLS*
}

\author{
BY \\ R. A. CLARK \\ AND \\ E. REISSNER \\ Case Institute of Technology \\ Massachusetts Institute of Technology
}

1. Introduction. In the present paper we consider a non-linear problem involving finite axi-symmetrical deflections of toroidal shells with circular cross section. The problem is that of an expansion joint for two straight sections of a cylindrical shell loaded in axial direction (Fig. 1). The particular kind of expansion joint investigated is."generally called an "Omega" joint.

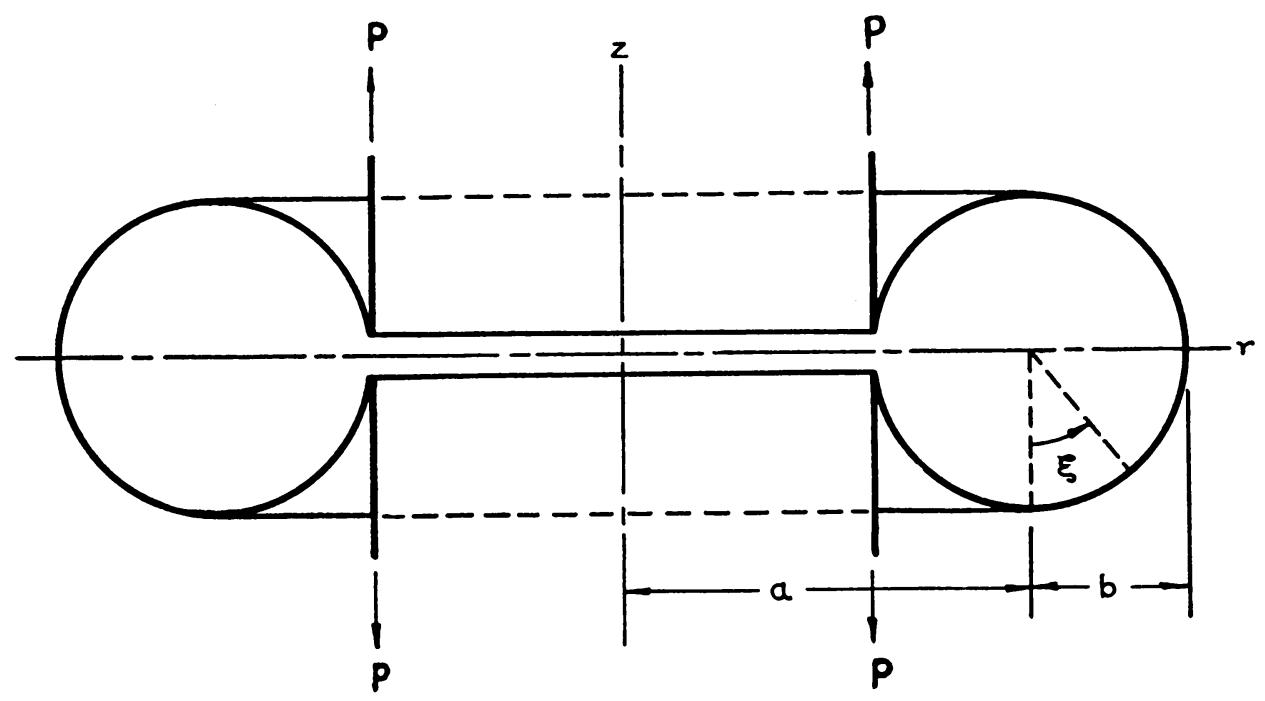

FIg. 1.

The linear theory of small deflections of such joints was recently considered by the first-named author [1]. The purpose of the present work is to establish the range of validity of the linearised theory and to obtain appropriate corrections to the linearised theory if the behaviour of the joint is slightly non-linear.

The results presented in what follows may, of course, be extended with relatively little modifications to other loading conditions for toroidal shells. The basic differential equations for finite axi-symmetrical bending of thin shells of revolution which are used in what follows may be found in a recent paper of the second-named author [2].

For the present problem, under assumptions which are discussed further on, we have to deal with the system of non-linear differential equations

$$
\begin{aligned}
& \beta^{\prime \prime}+\mu \psi \sin \xi=\mu \Omega(\cos \xi+\beta \sin \xi)+\mu \psi \beta \sin \xi, \\
& \psi^{\prime \prime}-\mu \beta \sin \xi=-\frac{1}{2} \mu \beta^{2} \cos \xi .
\end{aligned}
$$

*Received January 2, 1952. The present paper is a report on work done under the sponsorship of the Office of Naval Research under Contract N5-ori-07834 with Massachusetts Institute of Technology. 
subject to the boundary conditions $\beta( \pm \pi / 2)=\psi( \pm \pi / 2)=0$. The constant parameters are $\mu=\left[12\left(1-\nu^{2}\right)\right]^{1 / 2}\left(b^{2} / a h\right)$ where $b$ is the radius of the cross section of the middle surface of the shell, $a$ is the radius of the center line of the torus, $h$ is the wall thickness of the shell and $\nu$ is Poisson's ratio; and $\Omega=\left[12\left(1-\nu^{2}\right)\right]^{1 / 2}\left(a P / E h^{2}\right)$ where $P$ is the axial load per unit of circumferential length and $E$ is the modulus of elasticity of the shell.

As for the linearised problem, it is found that the following two ranges should be considered separately:

$$
\text { (i) } \mu=O(1) \text {; (ii) } \mu \gg 1 \text {. }
$$

When $\mu=O$ (1) the linearised theory is applicable as long as $\mu \Omega \ll 1$.

When $\mu \gg 1$, which is the case for many practical applications, the linearised theory is applicable as long as $\mu^{2 / 3} \Omega \ll 1$.

In these two cases non-linear corrections to the results of the linearised theory may be obtained by developments in powers of $\mu \Omega$ and $\mu^{2 / 3} \Omega$, respectively. The present paper contains numerical results for the second, less simple, of the two cases.

The physical significance of these results may be seen by considering the relative axial deflection $\delta$ of the two edges of the toroidal joint. As long as $\mu=O$ (1) we have $\delta=O(b \mu \Omega)=O\left(P b^{3} / E h^{3}\right)$ and the condition for linearity is $\delta \ll b$. When $\mu \gg 1$ we have $\delta=O(b \Omega)=O\left(P b a / E h^{2}\right)$ and the condition for linearity may be written in the form $\delta \ll b / \mu^{2 / 3}$. In the former case the relevant order of magnitude conditions are the same as those which hold if instead of a toroidal shell with center line radius $a$ we had a straight cylindrical shell with $a=\infty$. In the latter case the finite radius $a$ affects the results and non-linearity must be considered for progressively smaller values of $\delta$ as $\mu$ becomes larger. In either case the deflection $\delta$ may be large compared to the wall thickness $h$ of the shell without the occurrence of non-linear effects.

2. Differential equations for finite axi-symmetrical bending of thin toroidal shells. Let

$$
r_{0}=a+b \sin \xi, \quad z_{0}=-b \cos \xi
$$

be the parametric equations of the undeformed middle surface of a toroidal shell of constant thickness $h$. The basic differential equations for finite bending, up to terms of second degree, are then [2; Eqs. III and IV],

$$
\begin{aligned}
\beta^{\prime \prime}+\frac{r_{0}^{\prime}}{r_{0}} \beta^{\prime}-\left[\left(\frac{r_{0}^{\prime}}{r_{0}}\right)^{2}-\nu \frac{r_{0}^{\prime \prime}}{r_{0}}\right] \beta-\left[\frac{3}{2} \frac{r_{0}^{\prime} z_{0}^{\prime}}{r_{0}^{2}}-\frac{\nu}{2} \frac{z_{0}^{\prime \prime}}{r_{0}}\right] \beta^{2} \\
=-\frac{b^{2}}{r_{0} D}\left\{\Psi \sin \xi-\left(r_{0} V\right) \cos \xi-\beta\left[\Psi \cos \xi+\left(r_{0} V\right) \sin \xi\right]\right\}, \\
\Psi^{\prime \prime}+\frac{r_{0}^{\prime}}{r_{0}} \Psi^{\prime}-\left[\left(\frac{r_{0}^{\prime}}{r_{0}}\right)^{2}+\nu \frac{r_{0}^{\prime \prime}}{r_{0}}\right] \Psi-\left[2 \frac{z_{0}^{\prime} r_{0}^{\prime}}{r_{0}^{2}}+\nu \frac{z_{0}^{\prime \prime}}{r_{0}}\right] \beta \Psi-\nu \frac{z_{0}^{\prime}}{r_{0}} \beta^{\prime} \Psi \\
=\frac{b^{2} C}{r_{0}}\left[\beta \sin \xi-\frac{1}{2} \beta^{2} \cos \xi\right]+\left[\frac{r_{0}^{\prime} z_{0}^{\prime}}{r_{0}^{2}}+\nu \frac{z_{0}^{\prime \prime}}{r_{0}}\right]\left(r_{0} V\right)+\nu \frac{z_{0}^{\prime}}{r_{0}}\left(r_{0} V\right)^{\prime} \\
+\left[\frac{\left(z_{0}^{\prime}\right)^{2}-\left(r_{0}^{\prime}\right)^{2}}{r_{0}^{2}}-\nu \frac{r_{0}^{\prime \prime}}{r_{0}}\right] \beta\left(r_{0} V\right)-\nu \frac{r_{0}^{\prime}}{r_{0}}\left[\beta\left(r_{0} V\right)\right]^{\prime} \\
-\frac{b}{r_{0}}\left(r_{0}^{2} p_{H}\right)^{\prime}-\nu \frac{b r_{0}^{\prime}+b z_{0}^{\prime} \beta}{r_{0}^{2}}\left(r_{0}^{2} p_{H}\right) .
\end{aligned}
$$


In equations (2) and (3), $\Psi$ is a stress function, $\beta$ the change in the meridian angle due to deformation, $p_{H}$ the horizontal component of surface load intensity and $V$ the vertical (axial) stress resultant given by

$$
r_{0} V=-\int(a+b \sin \xi) b p_{V} d \xi
$$

where $p_{V}$ is the vertical component of surface load in intensity (Fig. 2a). The quantities $D$ and $C$ are defined by

$$
D=\frac{E h^{3}}{12\left(1-\nu^{2}\right)}, \quad C=E h .
$$

Primes indicate differentiation with respect to $\xi$.

In terms of $\Psi$ and $\beta$ we have, for the horizontal (radial) stress resultant $H$

$$
H=\Psi / r_{0},
$$

and for meridional and circumferential stress resultant $N_{\xi}$ and $N_{\theta}$, as indicated in Fig. 2b,

$$
\begin{aligned}
r_{0} N_{\xi} & =\Psi \cos \xi+\left(r_{0} V\right) \sin \xi+\beta\left[\Psi \sin \xi-\left(r_{0} V\right) \cos \xi\right], \\
b N_{\theta} & =\Psi^{\prime}+b r_{0} p_{H} .
\end{aligned}
$$

The meridional and circumferential stress couples $M_{\xi}$ and $M_{\theta}$ are given by

$$
\begin{aligned}
& b M_{\xi}=D\left[\beta^{\prime}+\nu\left(\frac{r_{0}^{\prime}}{r_{0}} \beta+\frac{1}{2} \frac{z_{0}^{\prime}}{r_{0}} \beta^{2}\right)\right], \\
& b M_{\theta}^{\prime}=D\left[\left(\frac{r_{0}^{\prime}}{r_{0}} \beta+\frac{1}{2} \frac{z_{0}^{\prime}}{r_{0}} \beta^{2}\right)+\nu \beta^{\prime}\right] .
\end{aligned}
$$

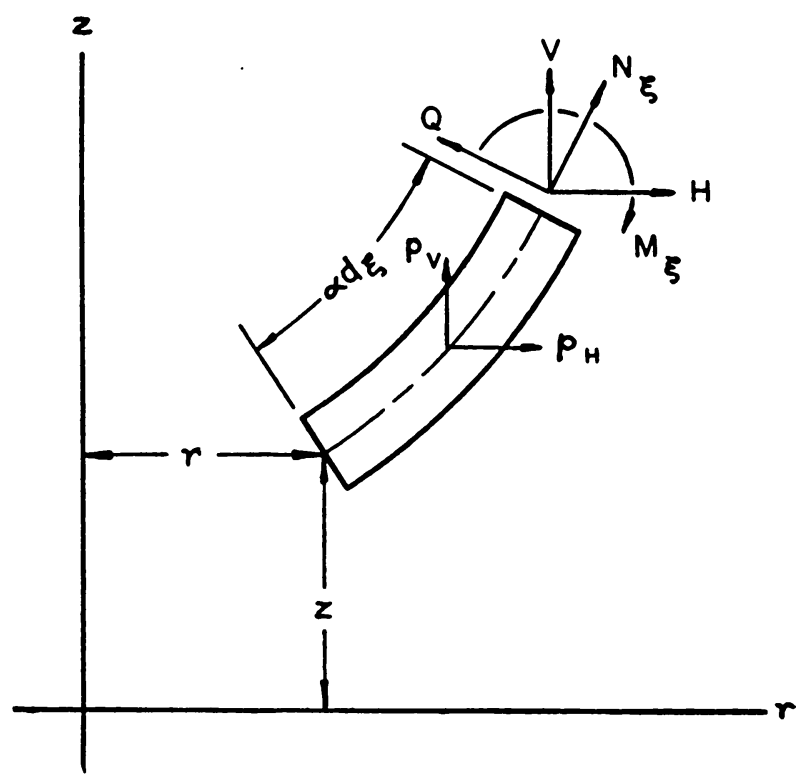

FIG. $2 a$. 


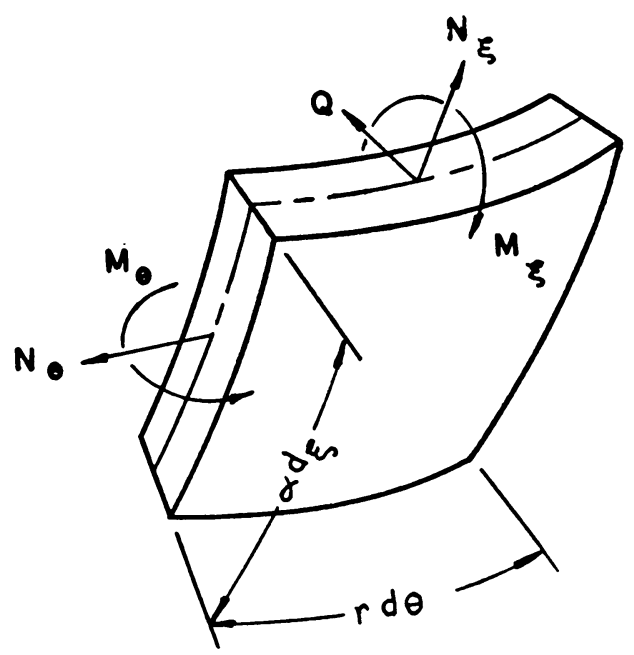

FIG. $2 b$.

The horizontal and vertical components of displacements $u$ and $w$ are given by

$$
\begin{aligned}
& u=\frac{r_{0}}{C}\left(N_{\theta}-\nu N_{\xi}\right), \\
& w=-b \int\left(\beta \cos \xi+\frac{1}{2} \beta^{2} \sin \xi\right) d \xi .
\end{aligned}
$$

Omission of all non-linear terms reduces equations (2) to (12) to the corresponding equations of the linearized theory which were considered in [1].

3. Dimensionless form of the differential equations for a shell subject to edge loads only. Let $P$ be the magnitude of the axial edge load per unit of circumferential length measured along the section $\xi=-\pi / 2$ of the toroidal shell and assume that no distributed surface loads are acting. This means that in equations (2) to (4)

and

$$
p_{H}=0, \quad p_{\mathrm{o}}=0
$$

$$
r_{0} V=a V_{0}=(a-b) P=\text { const. }
$$

As in the linearized theory one may define two parameters $\lambda$ and $\mu$ by

$$
\lambda=b / a, \quad \mu=\left[12\left(1-\nu^{2}\right)\right]^{1 / 2}\left(b^{2} / a h\right),
$$

and introduce a dimensionless stress function $\psi$ and a dimensionless load function $\Omega$ in the form

$$
\psi=\frac{\left[12\left(1-\nu^{2}\right)\right]^{1 / 2}}{E h^{2}} \Psi, \quad \Omega=\frac{\left[12\left(1-\nu^{2}\right)\right]^{1 / 2}}{E h^{2}} a V_{0}
$$

With this the differential equations (2) and (3) assume the form

$$
\begin{aligned}
\beta^{\prime \prime}+\frac{\cos \xi}{1+\lambda \sin \xi} \beta^{\prime}- & {\left[\left(\frac{\lambda \cos \xi}{1+\lambda \sin \xi}\right)^{2}+\frac{\nu \lambda \sin \xi}{1+\lambda \sin \xi}\right] \beta } \\
& -\left[\frac{3}{2} \frac{\lambda^{2} \sin \xi \cos \xi}{(1+\lambda \sin \xi)^{2}}-\frac{1}{2} \frac{\nu \lambda \cos \xi}{1+\lambda \sin \xi}\right] \beta^{2} \\
= & -\frac{\mu}{1+\lambda \sin \xi}[\psi(\sin \xi-\beta \cos \xi)-\Omega(\cos \xi+\beta \sin \xi) \bar{j}
\end{aligned}
$$




$$
\begin{aligned}
& \psi^{\prime \prime}+\frac{\lambda \cos \xi}{1+\lambda \sin \xi} \psi^{\prime}-\left[\left(\frac{\lambda \cos \xi}{1+\lambda \sin \xi}\right)^{2}-\frac{\nu \lambda \sin \xi}{1+\lambda \sin \xi}\right] \psi \\
&- {\left[2 \frac{\lambda^{2} \sin \xi \cos \xi}{(1+\lambda \sin \xi)^{2}}+\frac{\nu \lambda \cos \xi}{1+\lambda \sin \xi}\right] \beta \psi-\frac{\nu \lambda \sin \xi}{1+\lambda \sin \xi} \beta^{\prime} \psi } \\
&=\frac{\mu}{1+\lambda \sin \xi}\left[\beta \sin \xi-\frac{1}{2} \beta^{2} \cos \xi\right] \\
&+\Omega\left[\frac{\lambda^{2} \sin \xi \cos \xi}{(1+\lambda \sin \xi)^{2}}+\frac{\nu \lambda \cos \xi}{1+\lambda \sin \xi}\right. \\
&\left.+\left\{\frac{\lambda^{2}\left(\sin ^{2} \xi-\cos ^{2} \xi\right)}{(1+\lambda \sin \xi)^{2}}+\frac{\nu \lambda \sin \xi}{1+\lambda \sin \xi}\right\} \beta-\frac{\nu \lambda \cos \xi}{1+\lambda \sin \xi} \beta^{\prime}\right] .
\end{aligned}
$$

We note that the definitions (15) for $\lambda$ and $\mu$ and the fact that $b / h \gg 1$ for a thin shell always imply that $\lambda \ll \mu$. In what follows we restrict our attention to problems where we also have

$$
\lambda \ll 1,
$$

which means that the radius of the cross section of the torus is small compared with the radius of the center line of the torus

Under the assumption that (19) holds the differential equations (17) and (18) can be simplified considerably so as to read

$$
\begin{aligned}
& \beta^{\prime \prime}+\mu \sin \xi \psi=\mu[\Omega(\cos \xi+\beta \sin \xi)+\psi \beta \cos \xi], \\
& \psi^{\prime \prime}-\mu \sin \xi \beta=-\frac{1}{2} \mu \beta^{2} \cos \xi .
\end{aligned}
$$

The following discussion will be based on these two non-linear simultaneous differential equations for $\beta$ and $\psi$. $^{*}$

In terms of $\beta$ and $\psi$, again under the assumption that $\lambda \ll 1$, we have for direct and bending stresses and for displacements the following formulas

$$
\begin{aligned}
\sigma_{\theta D} & =\frac{N_{\theta}}{h}=\frac{E}{\left[12\left(1-\nu^{2}\right)\right]^{1 / 2}} \frac{h}{b} \psi^{\prime}, \\
\sigma_{\xi D} & =\frac{N_{\xi}}{h}=\frac{E}{\left[12\left(1-\nu^{2}\right)\right]^{1 / 2}} \frac{h}{a}[(\cos \xi+\beta \sin \xi) \psi+(\sin \xi-\beta \cos \xi) \Omega], \\
\sigma_{\xi B} & =\frac{6 M_{\xi}}{h^{2}}=\frac{E}{2\left(1-\nu^{2}\right)} \frac{h}{b} \beta^{\prime}, \quad \sigma_{\theta B}=\nu \sigma_{\xi B}, \\
\frac{u}{b} & =\frac{1}{\left[12\left(1-\nu^{2}\right)\right]^{1 / 2}} \frac{a h}{b^{2}} \psi^{\prime}=\frac{1}{\mu} \psi^{\prime}, \\
\frac{w}{b} & =-\int\left(\beta \cos \xi+\frac{1}{2} \beta^{2} \sin \xi\right) d \xi .
\end{aligned}
$$

4. Boundary conditions for problem of Omega joint. Since the solution of the problem in accordance with Fig. 1 is symmetrical with respect to the plane $z=0(\xi= \pm \pi / 2)$ we need consider only the lower half of the toroidal shell $(-\pi / 2 \leq \xi \leq \pi / 2)$. At the outside circumference of the torus $(\xi=\pi / 2)$, we have the symmetry conditions of no

*Equations (21) and (21) may be considered as equations for the leading terms in a development in powers of $\lambda$ of the solutions of the complete equations (17) and (18). 
change of slope of meridian curve due to deformation and of vanishing horizontal stress resultant $H$. In terms of $\beta$ and $\psi$ these conditions read

$$
\beta(\pi / 2)=0, \quad \psi(\pi / 2)=0 .
$$

At the inner edge of the torus $(\xi=-\pi / 2)$ we have conditions of elastic support through the two cylindrical shells to which the toroidal shell is joined. The complete formulation of these conditions is required when stresses and deformations are to be determined for small and moderate values of $\mu$. Our previous work [1] on the basis of linearised theory indicates that when $\mu \gg 1$ the exact form of these boundary conditions is unimportant for the determination of significant stresses and deformations.

Since explicit calculations in what follows are restricted to the case $\mu \gg 1$ the remaining boundary conditions are taken in the form

$$
\beta(-\pi / 2)=0, \quad \psi(-\pi / 2)=0 .
$$

5. Range of applicability of linearised theory for moderate values of parameter $\mu$ and method for determination of non-linear corrections. Inspection of the differential equations (20) and (21) indicates a difference in the character of the solutions depending on whether $\mu$ is of order of magnitude unity or is large compared to unity.

In the former case, that is when

$$
\mu=O(1),
$$

the only parameter which can become large is the load parameter $\mu \Omega$. This possibility, however, is found to be excluded by the physical conditions of the expansion joint problem. As long as (29) is satisfied we have the order of magnitude relations

$$
\beta=O(\mu \Omega), \quad \psi=O(\Omega) .
$$

From this the order of magnitude of the deflection $w$ is

$$
w=O(b \mu \Omega)
$$

Since $w=O(b)$, at most, it follows that $\mu \Omega=O(1)$ is the practically significant range.

Introduction of (30) into (20) and (21) indicates further that the linearised theory is applicable as long as

$$
\mu \Omega \ll 1
$$

or, in view of (31), as long as the deflections are small compared with the radius $b$ of the cross section of the torus, independent of the thickness $h$ of the shell.

Corrections to the linearised theory may be determined by developing the solutions of (20) and (21) in powers at the parameter

If we write

$$
\mu \Omega=\Lambda .
$$

$$
\beta=\mu \Omega \sum_{n=0}^{\infty} \Lambda^{n} \beta_{n}, \quad \psi=\Omega \sum_{n=0}^{\infty} \Lambda^{n} \psi_{n}
$$

and substitute these expansions into equations (20) and (21) we obtain

$$
\begin{gathered}
\sum_{n=0}^{\infty}\left(\beta_{n}^{\prime \prime}+\sin \xi \psi_{n}\right) \Lambda^{n}=\cos \xi+\Lambda\left(\sin \xi+\sum_{n=0}^{\infty} \psi_{n} \Lambda^{n} \cos \xi\right) \sum_{n=0}^{\infty} \beta_{n} \Lambda^{n}, \\
\sum_{n=0}^{\infty}\left(\psi_{n}^{\prime \prime}-\mu^{2} \sin \xi \beta_{n}\right) \Lambda^{n}=-\frac{1}{2} \mu^{2} \Lambda\left(\sum_{n=0}^{\infty} \beta_{n} \Lambda^{n}\right)^{2} \cos \xi .
\end{gathered}
$$


Equating coefficients of like powers of $\Lambda$ then gives the following pairs of equations for $\beta_{n}$ and $\psi_{n}$ :

$$
\begin{gathered}
\beta_{0}^{\prime \prime}+\sin \xi \psi_{0}=\cos \xi, \\
\psi_{0}^{\prime \prime}-\mu^{2} \sin \xi \beta_{0}=0, \\
\beta_{n}^{\prime \prime}+\sin \xi \psi_{n}=\beta_{n-1} \sin \xi+\sum_{k=0}^{n-1} \beta_{k} \psi_{n-k-1} \cos \xi, \quad n \geq 1, \\
\psi_{n}^{\prime \prime}-\mu^{2} \sin \xi \beta_{n}=\frac{1}{2} \mu^{2} \sum_{k=0}^{n-1} \beta_{k} \beta_{n-k-1} \cos \xi, \quad n \geq 1,
\end{gathered}
$$

where the first pair (37) are the equations for the linearised theory.

For sufficiently small values of $\mu$ each successive pair of equations (37) and (38) may be solved by developing $\beta_{n}$ and $\psi_{n}$ in powers of the parameter $\mu$ as

$$
\beta_{n}=\sum_{m=0}^{\infty} \beta_{n m} \mu^{m}, \quad \psi_{n}=\sum_{m=0}^{\infty} \psi_{n m} \mu^{m} .
$$

For moderate values of $\mu$ another approach is to determine solutions of (37) and (38) as Fourier series in a manner similar to that used for Mathieu functions.

As our work here is primarily intended to furnish an analysis valid when $\mu \gg 1$ we shall not carry out any explicit calculations for the case $\mu=O(1)$.

6. Approximate solutions for large values of $\mu$. We now consider the system of differential equations (20) and (21) with the boundary conditions (27) and (28) under the assumption that

$$
\mu \gg 1 \text {. }
$$

Our approach is guided to some extent by what we know [1] concerning the solution of the linearised problem under the assumption $\mu \gg 1$. For the linearised problem we have a narrow zone around $\xi=0$ where bending is important. In the remainder of the toroidal shell bending is a secondary effect and membrane action dominates. We shall show that an approximate solution for the non-linear problem may be found which is qualitatively similar to the solution of the linearised problem.

As long as we exclude the point $\xi=0$ we may obtain formal solutions of (20) and (21) by expanding as follows

$$
\beta=\sum_{n=0}^{\infty} \beta_{n} \mu^{-n}, \quad \psi=\sum_{n=0}^{\infty} \psi_{n} \mu^{-n} .
$$

Requiring the coefficients of all powers of $\mu$ to vanish after substitution of (41) in (20) and (21) we have the following relations

$$
\begin{aligned}
& \beta_{0}\left(\beta_{0}-2 \tan \xi\right)=0, \\
& \psi_{0}=\Omega \cot \xi+\beta_{0} \psi_{0} \cot \xi+\Omega \beta_{0}, \\
& \beta_{n}=\psi_{n-1}^{\prime \prime} \csc \xi+\frac{1}{2} \cot \xi \sum_{k=0}^{n} \beta_{k} \beta_{n-k}, \\
& \left.\psi_{n}=-\beta_{n-1}^{\prime \prime} \csc \xi+\Omega \beta_{n}+\cot \xi \sum_{k=0}^{n} \beta_{k} \psi_{n-k} .\right\}, \quad n_{-} \geq 1 \text {. }
\end{aligned}
$$


From (42) we have, in view of the boundary conditions (27) and (28),

$$
\beta_{0}=0, \quad \psi_{0}=\Omega \cot \xi,
$$

which is the solution of linearised membrane theory. With (44) we have next from (43)

$$
\beta_{1}=\psi_{0}^{\prime \prime} \csc \xi, \quad \psi_{1}=\Omega \beta_{1}+\psi_{0} \beta_{1} \cot \xi
$$

or

$$
\beta_{1}=2 \Omega \csc ^{3} \xi \cot \xi, \quad \psi_{1}=2 \Omega^{2} \csc ^{5} \xi \cot \xi .
$$

In order to have the first two non-vanishing terms in the expansions both for $\psi$ and $\beta$ we also determine $\beta_{2}$, with the result that

$$
\begin{aligned}
& \beta=2 \frac{\Omega}{\mu} \frac{\cos \xi}{\sin ^{4} \xi}\left[1+\frac{\Omega}{\mu}\left(17+43 \frac{\cos ^{2} \xi}{\sin ^{2} \xi}\right) \frac{1}{\sin ^{3} \xi}+\cdots\right], \\
& \psi=\Omega \frac{\cos \xi}{\sin \xi}\left[1+2 \frac{\Omega}{\mu} \frac{1}{\sin ^{5} \xi}+\cdots\right] .
\end{aligned}
$$

We may readily see that the complete expansions (47) satisfy the boundary conditions (27) and (28) term by term but their singularities at $\xi=0$ indicate that these expansions cannot be valid near $\xi=0$.

In order to obtain a solution which is valid near $\xi=0$ we observe that for $|\xi| \ll 1$ we may approximate (20) and (21) by

$$
\begin{aligned}
& \beta^{\prime \prime}+\mu \xi \psi=\mu[\Omega(1+\beta \xi)+\psi \beta], \\
& \psi^{\prime \prime}-\mu \xi \beta=-\frac{1}{2} \mu \beta^{2} .
\end{aligned}
$$

As for the linearised form of these equations we make the substitutions

$$
\begin{gathered}
x=\mu^{1 / 3} \xi, \\
\beta=\mu^{1 / 3} \Omega f(x), \quad \psi=\mu^{1 / 3} \sqrt{2} g(x)
\end{gathered}
$$

which transforms (50) into

$$
\begin{aligned}
& f^{\prime \prime}+x g=1+\mu^{2 / 3} \Omega f g+\Omega x f, \\
& g^{\prime \prime}-x f=-\frac{1}{2} \mu^{2 / 3} \Omega f^{2},
\end{aligned}
$$

where now primes indicate differentiation with respect to $x$.

We shall here restrict further attentions to problems for which

$$
\mu^{2 / 3} \Omega=O(1) .
$$

Equation (52) evidently must hold in order that the solution of the non-linear system (51) should have the same qualitative behaviour as the linearised system.

Since $\mu \gg 1$ we may now neglect the last term on the right of the first of equations (53). Setting further

$$
\Gamma=\mu^{2 / 3} \Omega,
$$


we are left with the system

$$
f^{\prime \prime}+x g=1+\Gamma f g, \quad g^{\prime \prime}-x f=-\frac{1}{2} \Gamma f^{2} .
$$

Since $\mu \gg 1$ we may replace the boundary conditions at $x= \pm\left(\pi_{/}^{\prime} 2\right) \mu^{1 / 3}$ by the following conditions

$$
f( \pm \infty)=0, \quad g( \pm \infty)=0 .
$$

We assume the solutions of (54) may be written in the form

$$
f=\sum_{n=0}^{\infty} \hat{f}_{n}(x) \Gamma^{n}, \quad g=\sum_{n=0}^{\infty} g_{n}(x) \Gamma^{n} .
$$

Substitution of the expansions (56) in (54) results in successive pairs of equations for the functions $f_{n}$ and $g_{n}$. Each pair of equations may be combined into one complex differential equation for a complex function $T_{n}$ defined by

$$
T_{n}(x)=f_{n}(x)+i g_{n}(x) .
$$

The resultant differential equations are

$$
T_{n}^{\prime \prime}(x)-i x T_{n}(x)=R_{n}(x), \quad n=0,1,2, \cdots
$$

where

$$
R_{0}(x)=1, \quad R_{n}(x)=\sum_{k=1}^{n} f_{k-1}\left[g_{n-k}-\frac{1}{2} i f_{n-k}\right], \quad n>1
$$

and where, in view of (55),

$$
T_{n}( \pm \infty)=0 .
$$

Two linearly independant solutions of the homogeneous equation corresponding to (58) are given by the tabulated functions $h_{1}(i x)$ and $h_{2}(i x)$ which are defined in terms of Hankel functions [3] by

$$
\begin{aligned}
& h_{1}(z)=\left(\frac{2}{3} z^{3 / 2}\right)^{1 / 3} H_{1 / 3}^{(1)}\left(\frac{2}{3} z^{3 / 2}\right), \\
& h_{2}(z)=\left(\frac{2}{3} z^{3 / 2}\right)^{1 / 3} H_{1 / 3}^{(2)}\left(\frac{2}{3} z^{3 / 2}\right) .
\end{aligned}
$$

The method of variation of parameters furnishes the solution of (58), (59) and (60) in the form

$$
T_{n}=-\frac{\pi}{4}\left(\frac{2}{3}\right)^{1 / 3}\left[h_{1}(i x) \int_{-\infty}^{x} R_{n}(t) h_{2}(i t) d t+h_{2}(i x) \int_{x}^{\infty} R_{n}(t) h_{1}(i t) d t\right],
$$

where use has been made of the fact that the Wronskian of $h_{1}(z)$ and $h_{2}(z)$ is $(4 / \pi i)(3 / 2)^{1 / 3}$.

The function $T_{0}$, which is a modified Lommel-function, and its first derivative have been tabulated previously [1]. In order to obtain an idea of the magnitude of the nonlinear effect we have calculated $T_{1}(x)$ and its first derivative by numerical integration 
using equation (62). Figures 3 and 4 contain graphs of the real and imaginary parts of the functions $T_{0}, T_{0}^{\prime}, T_{1}$ and $T_{1}^{\prime}$.

From the behaviour of the functions $h_{1}$ and $h_{2}$ it may be deduced that $f_{n}(x)$ is even in $x$ when $n$ is even and odd in $x$ when $n$ is odd while the reverse is true for $g_{n}(x)$.

We note further that from the asymptotic behaviour of the functions $h_{1}$ and $h_{2}$ it can be concluded that the behaviour of $T_{0}$ and $T_{1}$ for large values of $x$ is as follows

$$
\begin{array}{rlrl}
f_{0}(x) & \sim \frac{2}{x^{4}}, & & g_{0}(x) \sim \frac{1}{x}, \\
f_{1}(x) \sim \frac{86}{x^{9}}, & & g_{1}(x) \sim \frac{2}{x^{6}} .
\end{array}
$$

It remains now to interpolate between the functions $\beta$ and $\psi$ as defined by (50) and (56) for small values of $\xi$ and the functions $\beta$ and $\psi$ as defined by (47) for values of $\xi$ around $\xi= \pm \pi / 2$. We shall carry out this interpolation for the first two terms in the series for $\beta$ and for $\psi$. Write in equations (47), representing the solutions of (20) and (21) for sufficiently large values of $\xi$,

$$
y=\mu^{1 / 3} \sin \xi
$$

giving

$$
\begin{aligned}
& \beta=\mu^{1 / 3} \Omega \cos \xi \frac{2}{y^{4}}\left[1-\Omega \frac{26}{y^{3}}+\mu^{2 / 3} \Omega \frac{43}{y^{5}} \cdots\right], \\
& \psi=\mu^{1 / 3} \Omega \cos \xi \frac{1}{y}\left[1+\mu^{2 / 3} \Omega \frac{2}{y^{5}}+\cdots\right] .
\end{aligned}
$$

We now compare this with the solution around $\xi=0$ which may be written, in view of (50), (53), and (56), in the form

$$
\begin{aligned}
& \beta=\mu^{1 / 3} \Omega\left[f_{0}(x)+\mu^{2 / 3} \Omega f_{1}(x)+\cdots\right], \\
& \psi=\mu^{1 / 3} \Omega\left[g_{0}(x)+\mu^{2 / 3} \Omega g_{1}(x)+\cdots\right] .
\end{aligned}
$$

For sufficiently large values of $x=\mu^{1 / 3} \xi$ we have from (63) and (64)

$$
\begin{aligned}
& \beta \sim \mu^{1 / 3} \Omega \frac{2}{x^{4}}\left[1+\mu^{2 / 3} \Omega \frac{43}{x^{5}}+\cdots\right], \\
& \psi \sim \mu^{1 / 3} \Omega \frac{1}{x}\left[1+\mu^{2 / 3} \Omega \frac{2}{x^{5}}+\cdots\right] .
\end{aligned}
$$

In order that we may have a continuous transition between the solutions (66) and the solution (67) we now modify the solutions (67) to read

$$
\begin{aligned}
& \beta=\mu^{1 / 3} \Omega\left[f_{0}(y)+\mu^{2 / 3} \Omega f_{1}(y)+\cdots\right], \\
& \psi=\mu^{1 / 3} \Omega\left[g_{0}(y)+\mu^{2 / 3} \Omega g_{1}(y)+\cdots\right] .
\end{aligned}
$$

Since $x \sim y$ when $\xi$ is small we have agreement between (69) and (67) in this region. 


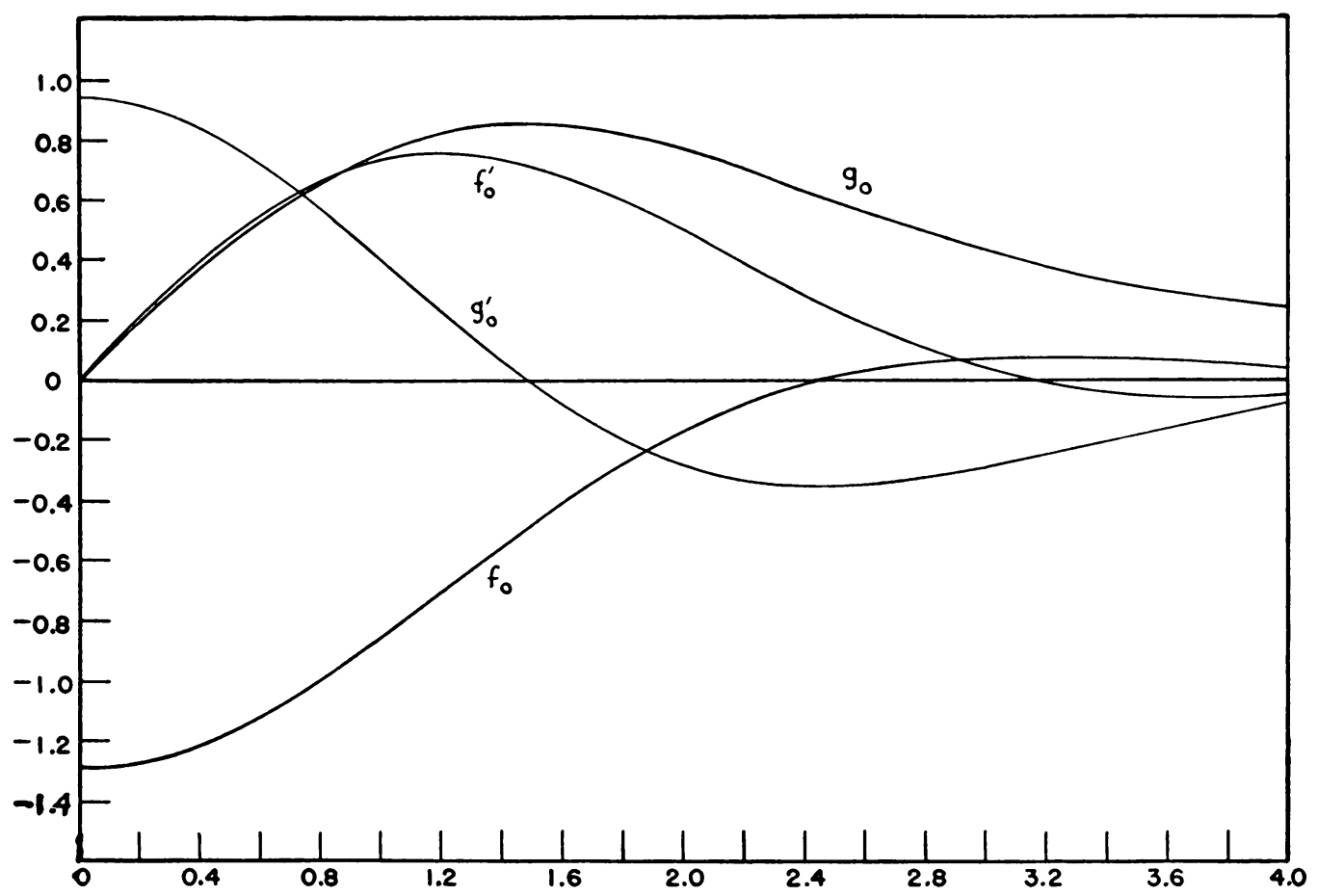

FIG. 3.

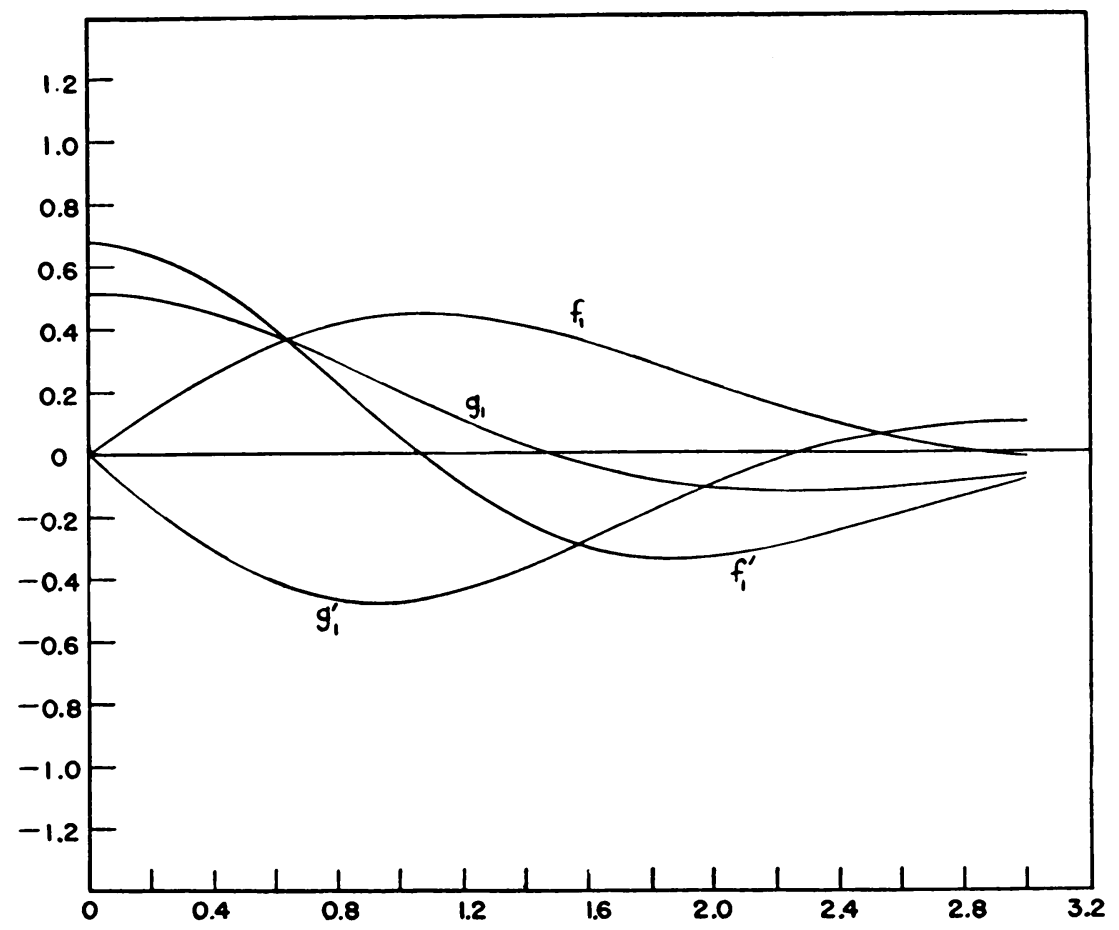

FIG. 4. 
For large $y$ equations (69) are, in view of (63) and (64)

$$
\begin{aligned}
& \beta \sim \mu^{1 / 3} \Omega \frac{2}{y^{4}}\left[1+\mu^{2 / 3} \Omega \frac{43}{y^{5}}+\cdots\right], \\
& \psi \sim \mu^{1 / 3} \Omega \frac{1}{y}\left[1+\mu^{2 / 3} \Omega \frac{2}{y^{5}}+\cdots\right] .
\end{aligned}
$$

Equations (70) agree with equations (66) in all terms except one. The reason for the absence of one of the terms in (70) which occurs in (66) is due to the fact that we omitted the last term in the first of equations (51). While this is permissible as long as $x$ is $O(1)$ it is no longer permissible for $|x| \gg 1$ and so we cannot expect complete matching of the developments (68) around $\xi=0$ and (67) around $\xi= \pm \pi / 2$. Since as we shall see all significant numerical contributions of the solutions are to be found in the vicinity of $\xi=0$ this lack of complete matching may be admitted without effective consequences.

7. Numerical results and determination of range of linearity for $\mu \gg 1$. In order to obtain an idea of the numerical effect of non-linearity for the expansion joint problem we must introduce the solutions (69) into the expressions (22) to (26) for stresses and displacements.

Just as in the linear problem the significant stresses are the circumferential direct. stress $\sigma_{\theta D}$ and the meridional bending stress $\sigma_{\xi B}$. These stresses have their largest values near the section $\xi=0$ and in the vicinity of this section we obtain from (22), (24) and (69)

$$
\begin{aligned}
\sigma_{\theta D} & =\frac{E}{\left[12\left(1-\nu^{2}\right)\right]^{1 / 2}} \frac{h}{b} \mu^{2 / 3} \Omega\left[g_{0}^{\prime}(y)+\mu^{2 / 3} \Omega g_{1}^{\prime}(y)+\cdots\right], \\
\sigma_{\xi B} & =\frac{E}{2\left(1-\nu^{2}\right)} \frac{h}{b} \mu^{2 / 3} \Omega\left[f_{0}^{\prime}(y)+\mu^{2 / 3} \Omega f_{1}^{\prime}(y)+\cdots\right],
\end{aligned}
$$

where $y \sim \mu^{1 / 3} \xi$.

The corresponding results of the linearised theory are equations (71) and (72) with only the first terms inside the brackets [1].

Since $g_{0}^{\prime}$ and $g_{1}^{\prime}$, and $f_{0}^{\prime}$ and $f_{1}^{\prime}$ are of the same order of magnitude (see Figures 3 and 4) we have in fact as the condition for negligible non-linear effects on stresses that the parameter $\mu^{2 / 3} \Omega$ must be sufficiently small.

According to the linearised theory $\sigma_{\theta D}$ is an even function of $\xi$ and has its maximum at $\xi=0$, while $\sigma_{\xi B}$ is an odd function of $\xi$ and has its maximum for $\xi=\xi_{m}$ where $f_{0}^{\prime \prime}\left(y_{m}\right)=0$ which relation is equivalent to $1-y_{m} g_{0}\left(y_{m}\right)=0$. The value of $y_{m}=$ $\mu^{1 / 3} \xi_{m}$ following from this relation was found in reference 1 to be $y_{m}= \pm 1.225$.

Inclusion of non-linear terms in (71) and (72) introduces both even and odd functions of $\xi$ into $\sigma_{\theta D}$ and $\sigma_{\xi B}$. The maximum of $\sigma_{\theta D}$ then no longer occurs for $y=0$. Let $y_{1}$ be the value of $y$ for which $\sigma_{\theta D}=\sigma_{\theta D, \max }$. . Equation (71) indicates that

$$
g_{0}^{\prime \prime}\left(y_{1}\right)+\mu^{2 / 3} \Omega g_{1}^{\prime \prime}\left(y_{1}\right)+\cdots=0
$$

or, in view of the form of the differential equations for $f$ and $g$, that

$$
2 y_{1}-\mu^{2 / 3} \Omega\left[f_{0}\left(y_{1}\right)+\mu^{2 / 3} \Omega f_{1}\left(y_{1}\right)+\cdots\right]=0 .
$$

Numerical values of $y_{1}$ for a few values of $\mu^{2 / 3} \Omega$ may be found in Table 1 , together with the corresponding values of $g^{\prime}\left(y_{1}\right)=g_{0}^{\prime}\left(y_{1}\right)+\mu^{2 / 3} \Omega g_{1}^{\prime}\left(y_{1}\right)$. It is seen that consideration 
of the first non-linear term increases the value of $\sigma_{\theta D, \max }$ as compared with the value of the quantity according to the linearised theory. This increase is, however, only slightly more than two percent up to values of 0.3 for the quantity $\mu^{2 / 3} \Omega$. The location of the maximum of $\sigma_{\theta D}$ is seen to be shifted toward the axis of the torus.

Maximum values $\sigma_{\xi B \text {, max }}$ of the bending stress $\sigma_{\xi B}$ occur according to (72) where $f_{0}^{\prime \prime}\left(y_{m}\right)+\mu^{2 / 3} \Omega f_{1}^{\prime \prime}\left(y_{m}\right)+\cdots=0$ or, in view of the differential equations (54) where

$$
\begin{aligned}
y_{m}\left[g_{0}\left(y_{m}\right)+\mu^{2 / 3} \Omega g_{1}\left(y_{m}\right)\right. & +\cdots] \\
& =1+\mu^{2 / 3} \Omega\left[f_{0}\left(y_{m}\right)+\mu^{2 / 3} \Omega f_{1}\left(y_{m}\right)\right]\left[g_{0}\left(y_{m}\right)+\mu^{2 / 3} \Omega g_{1}(y)\right] \\
& =1+\mu^{2 / 3} \Omega\left[f_{0}\left(y_{m}\right) g_{0}\left(y_{m}\right)\right]+\cdots
\end{aligned}
$$

Equation (74) has two solutions $y_{m, 1}$ and $y_{m, 2}$. Values of $y_{m, 1}$ and $y_{m, 2}$ may also be found in Table 1. It is seen that with increasing values of $\mu^{2 / 3} \Omega$ both of the locations of extremum values of $\sigma_{\xi B}$ shift towards the axis of the torus. In order to see the magnitude of the non-linear effect on $\sigma_{\xi B, \max }$ we have also tabulated values of $f^{\prime}\left(y_{m}\right)=f_{0}^{\prime}\left(y_{m}\right)+$ $\mu^{2 / 3} \Omega f_{1}^{\prime}\left(y_{m}\right)$. Non-linearity is seen to increase the bending stress at the inside maximum location and to decrease it at the outside maximum location. When $\mu^{2 / 3} \Omega=0.3$ the increase in stress is about six percent. When $\Omega$ is negative rather than positive this situation is reversed.

Table 1: Data for Values and Location of Maximum Stress

\begin{tabular}{lcccccc}
\hline \hline$\mu^{2 / 3 \Omega}$ & $y_{1}$ & $g^{\prime}\left(y_{1}\right)$ & $y_{m, 1}$ & $f^{\prime}\left(y_{m, 1}\right)$ & $y_{m, 2}$ & $f^{\prime}\left(y_{m, 2}\right)$ \\
\hline 0 & 0 & 0.939 & -1.225 & -0.753 & 1.225 & 0.753 \\
0.1 & -0.064 & 0.942 & -1.284 & -0.767 & 1.155 & 0.744 \\
0.2 & -0.130 & 0.949 & -1.337 & -0.784 & 1.080 & 0.741 \\
0.3 & -0.200 & 0.969 & -1.382 & -0.804 & 1.000 & 0.743 \\
\hline
\end{tabular}

Let $\delta$ be the relative axial displacement of the inner edges of the toroidal joint. We then have from (26)

$$
\frac{1}{2} \delta=w\left(\frac{\pi}{2}\right)-w\left(-\frac{\pi}{2}\right)=-b \int_{-\pi / 2}^{\pi / 2}\left(\beta \cos \xi+\frac{1}{2} \beta^{2} \sin \xi\right) d \xi .
$$

With $\beta$ from (69) and with $y=\mu^{1 / 3} \sin \xi$ equation (75) becomes

$$
\begin{aligned}
& \frac{1}{2} \delta=-b \int_{-\mu^{1 / \bullet}}^{\mu^{1 / \diamond}}\left\{\mu^{1 / 3} \Omega f(y) \frac{d y}{\mu^{1 / 3}}+\frac{1}{2} \mu^{2 / 3} \Omega^{2}[f(y)]^{2} \frac{y}{\mu^{1 / 3}} \frac{d y}{\mu^{1 / 3}\left\{1-\left(y / \mu^{1 / 3}\right)^{2}\right\}^{1 / 2}}\right\} \\
&=-\Omega b \int_{-\mu^{1 / \bullet}}^{\mu^{1 / \diamond}}\left[\left(f_{0}(y)\right.\right.\left.+\mu^{2 / 3} \Omega f_{1}(y)+\cdots\right) \\
&\left.+\frac{\Omega y}{2\left\{1-\left(y / \mu^{1 / 3}\right)^{2}\right\}^{1 / 2}}\left(f_{0}(y)+\mu^{2 / 3} \Omega f_{1}(y)+\cdots\right)^{2}\right] d y .
\end{aligned}
$$

Since $f_{0}(y)$ is even in $y$ and $f_{1}(y)$ odd in $y$ we see from (76) that

$$
\frac{1}{2} \delta=-\Omega b\left[\int_{-\mu^{1 / s}}^{\mu^{1 / 2}} f_{0}(y) d y+O\left(\mu^{4 / 3} \Omega^{2}\right)\right] .
$$


The leading term in the omitted expression is due to the function $f_{2}(y)$ which has not yet been calculated. Since $f_{1}$, which gave first order corrections for the stresses, does not contribute to the value of $\delta$, we may conclude that non-linear corrections to $\delta$ are, for small values of $\mu^{2 / 3} \Omega$, even less significant than are these corrections for the values of the stresses. We know [1] that

$$
\int_{-\mu^{1 / 2}}^{\mu^{1 / 0}} f_{0}(y) d y \sim \int_{-\infty}^{\infty} f_{0}(y) d y=-\pi
$$

and so

$$
\delta=2 \pi \Omega b\left[1+O\left(\mu^{4 / 3} \Omega^{2}\right)\right] .
$$

From (79) we may obtain the order of magnitude of the relative axial displacement up to which the linearised theory is applicable. The condition for linearity $\mu^{2 / 3} \Omega \ll 1$ may, from (79), be written in the form

$$
\delta \ll \frac{2 \pi b}{\mu^{2 / 3}}=\frac{2 \pi}{3\left[12\left(1-\nu^{2}\right)\right]^{1 / 2}} \frac{(a b)^{2 / 3}}{b^{1 / 3}} .
$$

For practical purposes we may take instead of (80)

$$
\delta<\frac{2 \pi b}{3 \mu^{2 / 3}} .
$$

The inequalities (80) are remarkable for the facts that (1) displacements much larger than the wall thickness of the shell fall within the scope of the linearised theory, and (2) the ratio of permissible displacement $\delta$ to the radius of the torus decreases as the parameter $\mu$ increases.

\section{REFERENCES}

1. R. A. Clark, On the theory of thin elastic toroidal shells, J. Math. Phys., 29, 146-178 (1950).

2. E. Reissner, On axisymmetrical deformation of thin shells of revolution, Proc. Symp. Appl. Math. 3, 27-52 (1950).

3. Tables of the modified Hankel functions of order one-third and their derivatives, Ann. Harv. Comp. Lab., Vol. 2, Cambridge, Mass., Harvard University Press, 1945. 